http://jmscr.igmpublication.org/home/

ISSN (e)-2347-176x ISSN (p) 2455-0450

crossref DOI: https://dx.doi.org/10.18535/jmscr/v7i7.09

\title{
A case of Mixed Connective Tissue Disease with Right Heart Failure
}

\author{
Authors \\ Dr Venkatesh $\mathbf{R}^{1^{*}}$, Dr Nagarajan $\mathrm{K}^{2}$, Dr Anand $\mathbf{P}^{3}$ \\ ${ }^{1}$ Post-Graduate, Department of General Medicine, Sri Manakula Vinayagar Medical College and Hospital \\ (SMVMCH), Puducherry 605107 \\ ${ }^{2}$ Professor and Chief, Department of General Medicine, SMVMCH \\ ${ }^{3}$ Assistant Professor, Department of General Medicine, SMVMCH \\ *Corresponding Author \\ Dr Venkatesh $\mathbf{R}$ \\ Final year post graduate, Department of General medicine
}

Room no 12, SMVMCH medical boys PG hostel, Sri Manakula Vinayagar Medical College and Hospital

(SMVMCH), Kalitheerthalkuppam, Puducherry 605107, India

\begin{abstract}
Background: Sharp and colleagues in 1972 was first described the Mixed connectivetissue disease (MCTD).They described MCTD in a group of people havingwith overlapping clinical features of scleroderma, myositis and systemic lupus erythematosus (SLE) with the presence of a specific antibody against U1-ribonucleoprotein (RNP). ${ }^{1}$ Sharp's syndrome is the other name for MCTD. ${ }^{2}$ It is more common in females than males. Estimations of the female to male ratio varies from approximately $3: 1$ to $16: 1{ }^{3,4}$ It is typically occuring between 15-25 years oldbut it can occur at any age but in our country has rarely been reported but here by reported rare presentation of MCTD occurs in 65 years old female.

Keywords: Mixed connective tissue disease; Pulmonary hypertension; Extractable nuclear antigen.
\end{abstract}

\section{Introduction}

Mixed connective tissue disease is the systemic inflammatory rheumatic disease very uncommon. If the patient has features of more than one classical inflammatory rheumatic disease then it is categorized under rheumatic "overlap syndromes". It is the specific subset of MCTD. Mixed connective tissue disease can occur in any age from childhood to adulthood. 37 years is the average age of disease onset. Approximately 75 percent of individuals affected are females. In Norway MCTD has been found to be 3.8 per 100,000 adults. $^{3}$ These classic rheumatic diseases includepolymyositis, scleroderma, rheumatoid arthritis and systemic lupus erythematosus. Laboratory result distinguishes MCTD from other overlap syndromes. Patients of MCTD have rheumatic overlap syndrome plus anti-RNP antibodies. ${ }^{5}$

\section{Case Report}

A 65 years old female, came with thecomplaints of difficulty in breathing and swallowing, palpitations, giddiness, swelling of hands, right shoulder pain, cough with expectoration, and oral dryness with co-morbidities such as Type II 
diabetes mellitus and hypertension. On general examination she was conscious, oriented with stable vitals, elevated JVP with "v" wave, and bilateral pitting pedal edema. Systemic examination revealed loud P2 present, pan systolic murmur in tricuspid area, and bilateral basal inspiratory crackles $(\mathrm{L}>\mathrm{R})$. she was further evaluated by algorithmic approach for pulmonary hypertension. Re-examination revealed difficulty in opening the mouth and tightening of the skin.Sinus tachycardia, and right ventricular hypertrophy was ECG findings. Moderate TR with severe $\mathrm{PAH}$, and dilated RA and RV was showed in 2D ECHO. HRCT of thorax showed nonspecific interstitial pneumonia. She had positive of ANA. (speckled pattern)-1:1000 (strong positive). Finally we did extractable nuclear antigen and it was positive for U1 RNP, Antibody IgG, Sm Antibody IgG.With these clinical and laboratory findings a diagnosis of mixed connective tissue diseasewas made. This patient was treated with immunosuppressives and supportive measures. The patient improved symptomatically and got discharged.Now she is on regular follow up.

\section{1)Algorithmic approach for PHTN}

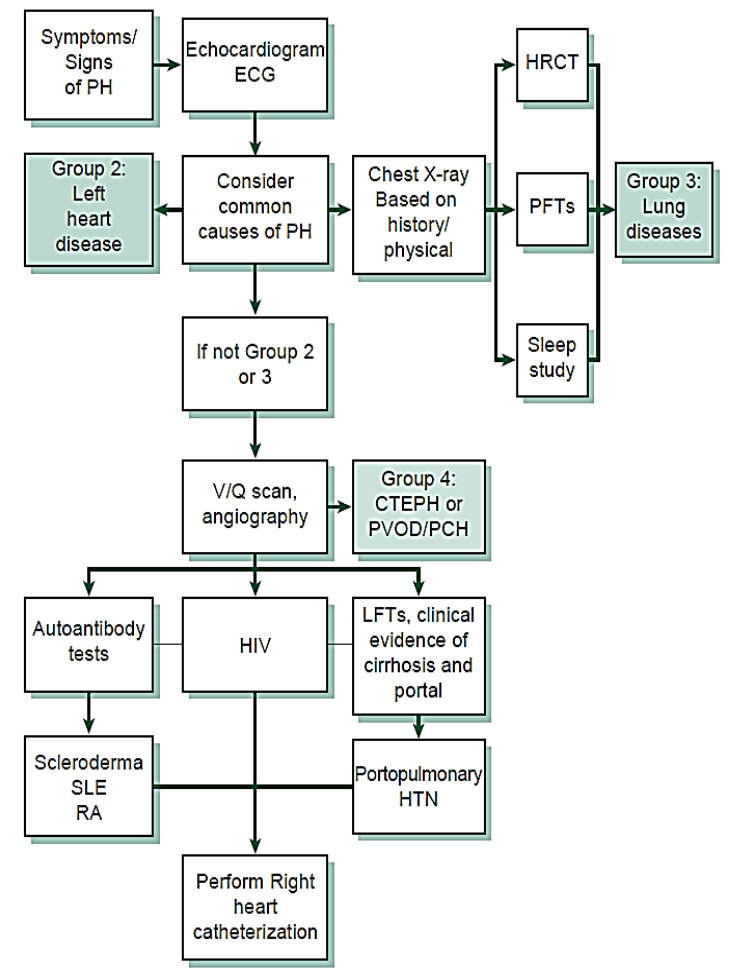

2)ECG shows

- Sinus tachycardia

- Right axis deviation

- RBBB

- Right ventricular hypertrophy

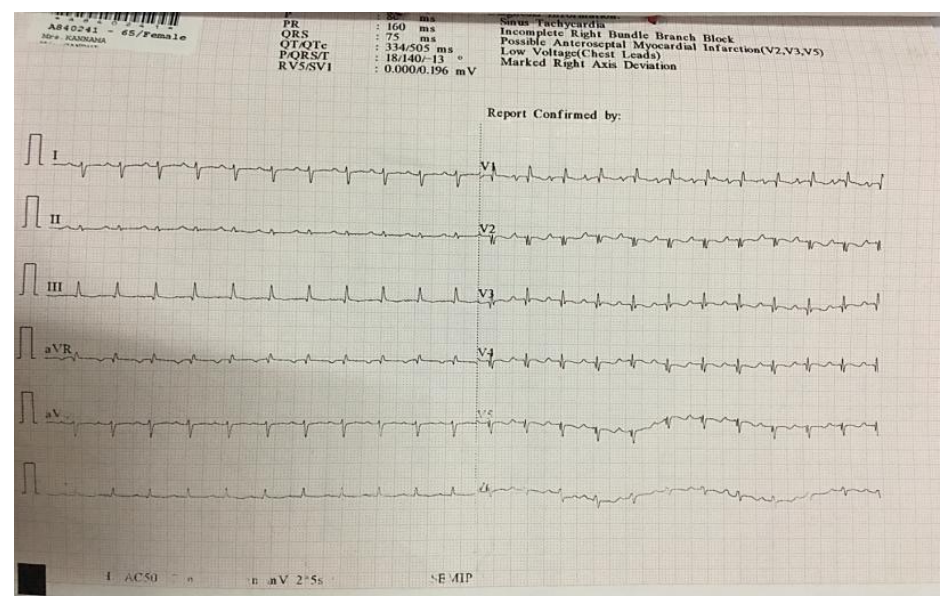

\section{3)Mask Like Facies}

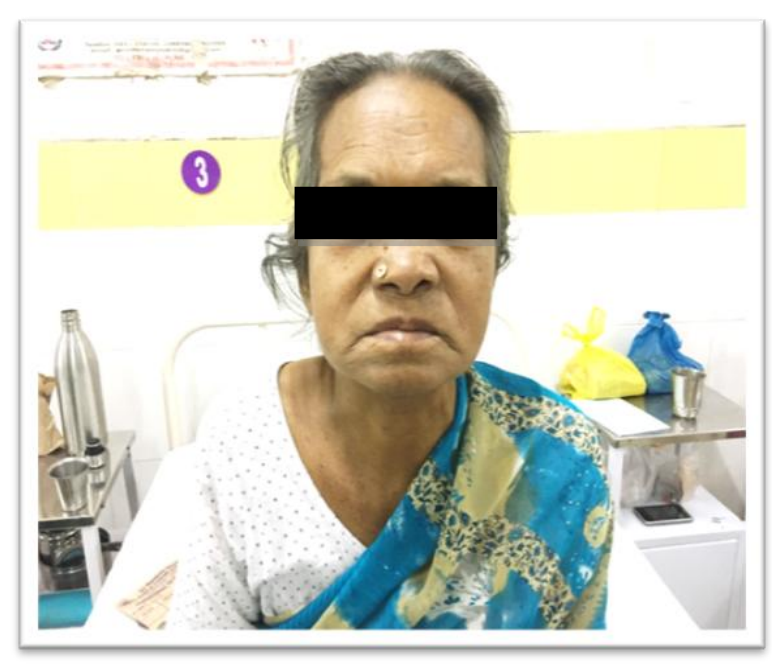

\section{4)Difficulty in Opening Mouth}

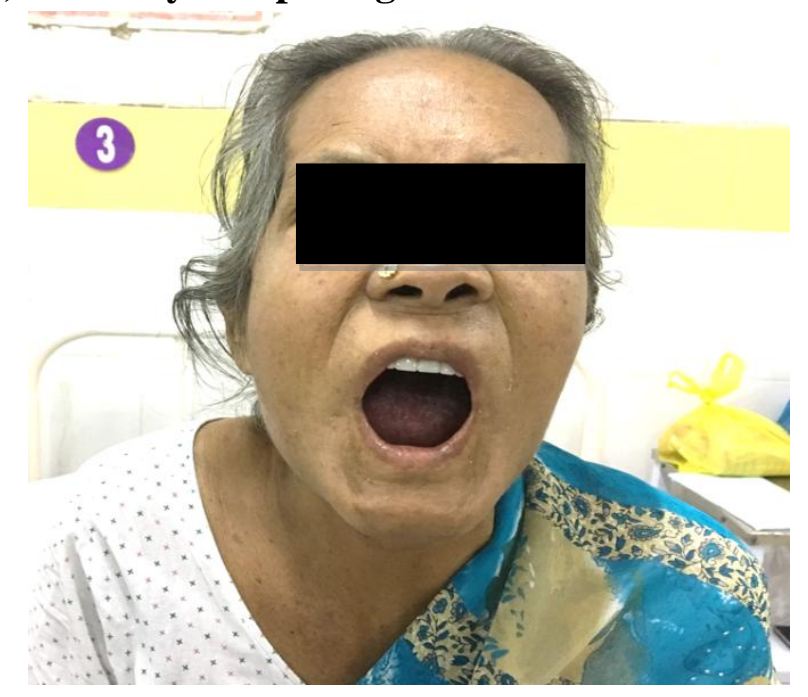




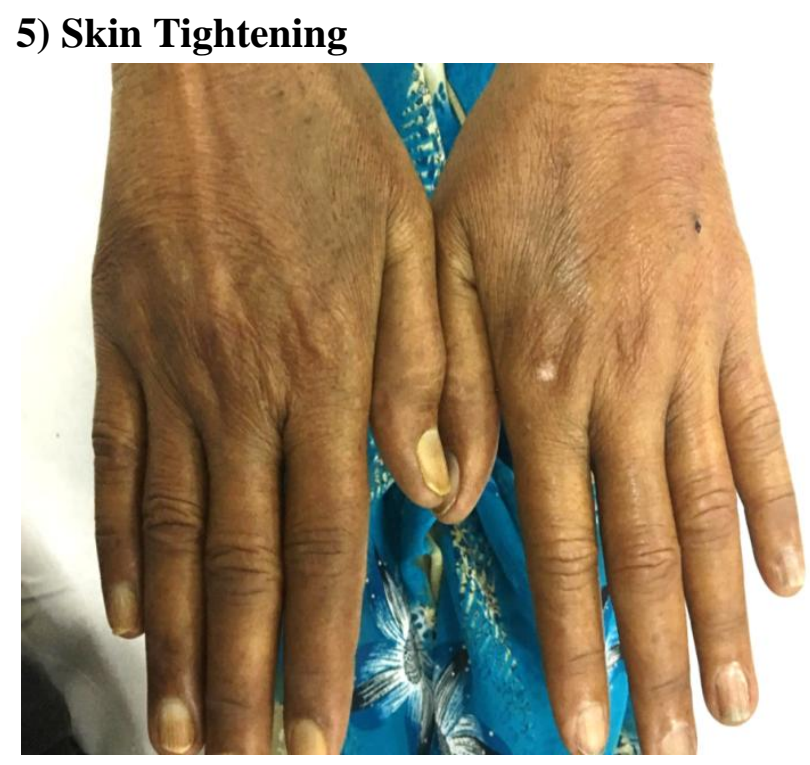

\section{6) HRCT Throax Shows Non Specific Interstitial Pneumonia}

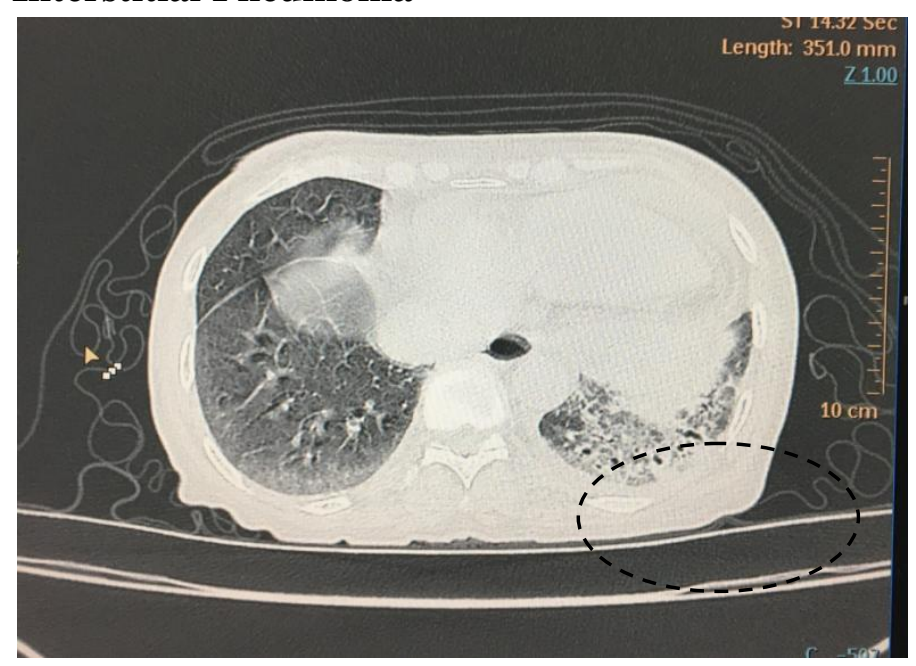

\section{Discussion}

Mixed connective tissue disease is systemic inflammatory rheumatic disease which is uncommon. It is also classified in the category of rheumatic "overlap syndromes". It is the term used to describes when features of more than one classic inflammatory rheumatic disease in patients of MCTD. ${ }^{5}$ These classic rheumatic diseases features include systemic lupus erythematosus, scleroderma, polymyositis, and rheumatoid arthritis.Patients with MCTD have rheumatic overlap syndrome plus anti-RNP antibodies. ${ }^{6}$

Raynaud's phenomenon may precede the development of additional symptoms of MCTD. It is also seen in scleroderma. Painful cold fingers and toes with blue or white color changes which is caused by spasm of blood vessels in the hands and feet in response to cold or stress is the characteristic features. 90 percent of individuals with MCTD will be having this features.

Majority of patients with MCTD is affected with pain in multiple joints (polyarthralgia) or inflammation of joints (arthritis). In sun exposed areas lupus like skin inflammationis common. Hair loss are common associated with skin scarring changes on the fingers and face like features seen in scleroderma. Muscle weakness due to inflammation (myositis) of proximal muscle groups can occur and also associated with some additional frequent symptoms include hand swelling and fatigue.

In at least half of patients with MCTD having features of esophagus dysfunction. It is often manifests as the symptoms of heartburn and difficulty in swallowing solid foods. Patient with features of lung disease may lead to breathing difficulties caused either by causing lung inflammation and scarring in and around the air sacs which causes interstitial lung disease or high blood pressure in the lungs will lead to pulmonary hypertension.

Heart involvement is less common in MCTD than lung problems but can be serious if it occurs. The incidence of kidney disease is less common when compared to lupus. 10 percent of individuals with MCTD approximately having neurologic abnormalities.

Mixed connective tissue disease may be diagnosed based upon a thorough clinical evaluation, detailed patient history, identification of characteristic findings, and abnormally high levels of antibodies to the U1 small nuclear ribonucleoprotein (anti-RNP) is the specialized blood test that helps in diagnosis.

\section{Treatment}

The treatment of mixed connective tissue disease is based on the specific symptoms that present in variouscases. The clinical experience by MCTD experts supports the use of antimalarial drugs for potential lupus like disease modifying effects the 
use of vasodilators to treat Raynaud's phenomenon.

In patients with GERDuse of proton pump inhibitors is helpful in relieving symptoms and additional disease modifying anti rheumatic drugs (DMARDs) for rheumatoid arthritis is also useful in treating the disease. ${ }^{7}$

A course of aggressive immunosuppression is needed for MCTD patients with pulmonary hypertension or other lung disease. Corticosteroids with low to moderate doseis more effective for rapid control of disease flares and it may be used as part of therapy as long term. Mild inflammatory symptoms can be controlled by Nonsteroidal anti inflammatory drugs (NSAIDs). It rarely can cause aseptic meningitis in some individuals. $^{7}$

\section{Conclusion}

In our country this type of cases are very rare. Death in these cases may be due to pulmonary hypertension. Hence these patients must be counseled and re assured.The main goal of therapy in mixed connective tissue diseaseis to maintain function and control symptoms. Medical therapy to specific organ involvement and extent of disease activity. Hence monitoring for development of complications such as pulmonary hypertension or infection is important in management.

\section{References}

1. Sharp GC, Irvin WS, Tan EM, Gould RG, Holman HR (February 1972). "Mixed connective tissue disease--an apparently distinct rheumatic disease syndrome associated with a specific antibody to an extractable nuclear antigen (ENA)". The American Journal of Medicine. 52 (2):148-59. doi:10.1016/00029343(72)90064-2. PMID 4621694.

2. Rapini RP, Bolognia JL, Jorizzo JL (2007). Dermatology: 2-Volume Set. St. Louis: Mosby. ISBN 1-4160-2999-0.
3. Nakae K, Furusawa F, Kasukawa R, et al.A nationwide epidemiological survey on diffuse collagen diseases: Estimation of prevalence rate in Japan. Kasukawa R, Sharp G, eds. Mixed Connective Tissue Disease and Anti-nuclear Antibodies. Amsterdam: Excerpta Medica; 1987. 9.

4. Gunnarsson R, Molberg O, Gilboe IM, Gran JT, PAHNOR1 Study Group. The prevalence and incidence of mixed connective tissue disease: a national multicentre survey of Norwegian patients. Ann Rheum Dis. 2011 Jun. 70 (6):1047-51.

5. Martinez-Barrio J, Valor L, Lopez-Longo FJ. Facts and controversies in mixed connective tissue disease. Med Clin (Barc). 2017 Aug 29.

6. Hoffman RW (1 June 2009). "Mixed Connective Disease". In Stone J. Pearls \& Myths in Rheumatology. Springer. pp. 169-172. ISBN 978-1-84800-933-2. Retrieved 26 June 2010.

7. Eric L Greidinger, MD Associate Professor, Department of Medicine, Division of Rheumatology and Immunology, University of Miami Miller School of Medicine, Miami Veterans Affairs Medical Center. 